\title{
Structural and Functional Plasticity within the Nucleus Accumbens and Prefrontal Cortex Associated with Time-Dependent Increases in Food Cue-Seeking Behavior
}

\author{
Paige M Dingess', Rebecca A Darling', Rifka C Derman², Shaun S Wulff ${ }^{3}$, Melissa L Hunter ${ }^{4}$, \\ Carrie R Ferrario ${ }^{2,5}$ and Travis E Brown ${ }^{*, 1,3}$ \\ 'Neuroscience Program, University of Wyoming, Laramie, WY, USA; ${ }^{2}$ Neuroscience Program, University of Michigan, Ann Arbor, MI, USA; \\ ${ }^{3}$ Department of Statistics, University of Wyoming, Laramie, WY, USA; ${ }^{4}$ School of Pharmacy, University of Wyoming, Laramie, WY, USA; \\ ${ }^{5}$ Department of Pharmacology, University of Michigan, Ann Arbor, MI, USA
}

\begin{abstract}
Urges to consume food can be driven by stimuli in the environment that are associated with previous food experience. Identifying adaptations within brain reward circuits that facilitate cue-induced food seeking is critical for understanding and preventing the overconsumption of food and subsequent weight gain. Utilizing electrophysiological, biochemical, and Dil labeling, we examined functional and structural changes in the nucleus accumbens (NAc) and prefrontal cortex (PFC) associated with time-dependent increases in food craving ('incubation of craving'). Rats self-administered 60\% high fat or chow $45 \mathrm{mg}$ pellets and were then tested for incubation of craving either I or 30 days after training. High fat was chosen for comparison to determine whether palatability differentially affected incubation and/or plasticity. Rats showed robust incubation of craving for both food rewards, although responding for cues previously associated with high fat was greater than chow at both I and 30 days. In addition, previous experience with high-fat consumption reduced dendritic spine density in the PFC at both time points. In contrast, incubation was associated with an increase in NAc spine density and $\alpha$-amino-3hydroxy-5-methyl-4-isoxazolepropionic acid receptor (AMPAR)-mediated transmission at 30 days in both groups. Finally, incubation of craving for chow and high fat was accompanied by an increase in calcium-permeable and calcium-impermeable AMPARs, respectively. Our results suggest that incubation of food craving alters brain reward circuitry and macronutrient composition specifically induces cortical changes in a way that may facilitate maladaptive food-seeking behaviors.
\end{abstract}

Neuropsychopharmacology (2017) 42, 2354-2364; doi:I0.1038/npp.2017.57; published online 12 April 2017

\section{INTRODUCTION}

Environmental stimuli can powerfully motivate behavior. With the massive increase in caloric intake in developed nations (Caballero, 2007), one behavior of special interest is that of feeding. From an evolutionary perspective, opportunities to consume food were often sporadic and it was therefore adaptive for humans to recognize and act upon cues predictive of food availability (Eknoyan, 2006). Unfortunately, overconsumption occurs when the availability of food exceeds the biological need and food-associated cues continue to trigger the instinct to consume.

Indeed, clinical studies have revealed that humans will overconsume food after attending to food-related cues, including visual presentation (Marcelino et al, 2001; Fisher and Birch, 2002; Coelho et al, 2009) and the smell of food

*Correspondence: Dr TE Brown, School of Pharmacy, Neuroscience Program, University of Wyoming, 1000 E. University Avenue, Department 3375, Laramie, WY 8207I, USA, Tel: 307766 6I29, Fax: 307766 2953, E-mail: tbrown53@uwyo.edu

Received 6 October 2016; revised 8 March 2017; accepted 9 March 2017; accepted article preview online 15 March 2017
(Fedoroff et al, 1997; Jansen et al, 2003; Jansen et al, 2008). Cue exposure can even induce overeating when the cue presented is not relevant to the food available (eg, seeing an advertisement for one food item can promote consumption of another; Harris et al, 2009). Cue-potentiated feeding is a behavior readily observed in rodents as well. Petrovich et al (2007) demonstrated that reintroduction to cues paired with specific food pellets when rats were hungry enhanced consumption of those pellets when the rats were subsequently satiated (Petrovich et al, 2007), suggesting that foodpaired cues, in addition to signaling the presence of food, may induce a state of 'craving'. Such motivational states can be altered by diet, as evidenced by studies indicating that obese rats exposed to 'junk-food' or palatable high-fat (HF) diets exhibit greater cue-triggered motivation (Brown et al, 2015; Robinson et al, 2015). These observations indicate that not only are food-predictive cues important for triggering food seeking and consumption in rodents but moreover that such cues are most salient in those that develop obesity. Thus, understanding the mechanisms underlying cueinduced seeking behavior may provide useful insights to the treatment of obesity and other overeating disorders. 
Efforts have been made to identify reward circuit regions that are altered by feeding. For example, consumption of a 'junk-food' diet induces an increase in calcium-permeable $\alpha$-amino-3-hydroxy-5-methyl-4-isoxazolepropionic acid receptors (CP-AMPARs; Oginsky et al, 2016) and HF diet-induced obesity elicits an increase in AMPA to $N$-methyl-D-aspartate (NMDA) ratio (Brown et al, 2015) in the nucleus accumbens core (NAc-C). In addition, previous work within our laboratory has shown that non-contingent consumption of a $60 \%$ HF diet induces a reduction of thintype spines in the infralimbic medial prefrontal cortex (IL-mPFC; Dingess et al, 2016). Behaviorally, we have shown that there is an increase in lever responding for foodassociated cues over time, independent of macronutrient composition (Darling et al, 2016). What remains unknown, and this manuscript begins to address, are the neuroadaptations that correspond with this enhanced time-dependent motivation for food cues. This is important to investigate given that unrewarded cue presentations gain salience over time, a phenomenon known as 'incubation of craving', and exposure to such cues is hypothesized to precede relapse behavior (O'Brien et al, 1992; Lu et al, 2004; Grimm et al, 2011), which in the context of feeding behavior, may manifest as a failure to maintain healthy eating habits.

The incubation of craving model has become a useful tool to evaluate cue responsiveness for many drugs of abuse (Grimm et al, 2001; Bienkowski et al, 2004; Lu et al, 2004; Shepard et al, 2004; Abdolahi et al, 2010) but has been observed for food as well, including sucrose pellets (Grimm et al, 2005; Grimm et al, 2011), liquid saccharin (Aoyama et al, 2014), HF pellets (Darling et al, 2016) and even food pellets with a similar macronutrient composition to standard lab chow (Krasnova et al, 2014; Darling et al, 2016). Briefly, it involves training an animal to press a lever and receive a reward (eg, food pellet) in conjunction with associated cues (eg, light and tone). Following a training period, the animal lever presses for the cues alone, in the absence of the reward. Incubation of craving occurs when the responsiveness towards these cues becomes greater over time. Investigation of this paradigm has led to important discoveries regarding the neural substrates of cue-potentiated reward seeking. Studies using a variety of techniques have implicated the PFC (Koya et al, 2009; Ma et al, 2014) and NAc (Hollander and Carelli, 2007; Wolf and Ferrario, 2010; Counotte et al, 2014) as regions, which undergo plasticity following incubation of craving. The NAc-C in particular has gained attention due to the extensive structural and functional glutamatergic plasticity that occurs following incubation of cocaine craving, including increases in spine density (Christian et al, 2016) and AMPA/NMDA ratio (Ortinski et al, 2012) as well as an upregulation in CP-AMPARs (Conrad et al, 2008). In contrast to drug reward, repeated exposure to natural rewards, such as sexual experience (Pitchers et al, 2012) or incubation of sucrose craving, induces a decrease in AMPA/ NMDA (Counotte et al, 2014).

With the exception of sucrose, the neural mechanisms underlying the incubation of food craving have not been investigated. Thus, we utilized behavioral, histological, electrophysiological, and biochemical analyses to examine structural and functional plasticity in primary reward circuit regions, the PFC and NAc, following incubation of craving for HF vs chow food rewards. The chow pellets were manufactured to mimic the macronutrient composition of the standard rodent chow for the purpose of evaluating the effect of incubated food craving more generally. HF was chosen for comparison to assess the influence that palatability might have on the observed neurophysiological adaptations, given that we have previously shown that these pellets are preferred over the chow pellets (Darling et al, 2016).

\section{MATERIALS AND METHODS}

\section{Animals}

For biochemical studies, male Sprague-Dawley rats were purchased from Harlan and for all other studies, bred in house. All animals were housed in a temperature-controlled room with a 12:12-h light-dark cycle. All animals had free access to food and water throughout. In all studies, rats were 60-70 days old at the start of experimentation. All procedures were approved by the UW or UM Institutional Animal Care and Use Committee.

\section{Diets}

All rats were maintained on standard lab chow in the home cage throughout experimentation (\#5001, Labdiet, St Louis, MO: $\sim 29 \%$ protein, $\sim 58 \%$ carbohydrate, and $\sim 13 \%$ fat by kilocalorie; $3.36 \mathrm{kcal} / \mathrm{g}$ ). During self-administration, rats either lever pressed for a custom chow pellet manufactured to closely match home-cage chow composition (\#F0165, BioServ, Flemington, NJ; 25.3\% protein, $64.5 \%$ carbohydrates, and $10.1 \%$ fat by kilocalorie; $3.35 \mathrm{kcal} / \mathrm{g}$ ), or $\mathrm{HF}$ pellets (\#F07062, Bio-Serv; 19.4\% protein, $60.8 \%$ fat, and $19.9 \%$ carbohydrates by kilocalorie; $3.72 \mathrm{kcal} / \mathrm{g}$ ).

\section{Food Self-Administration}

Chow or HF self-administration was conducted in standard operant boxes (Med Associates, St Albans, VT; $30.5 \mathrm{~cm} \times 24.1 \mathrm{~cm} \times 21.0 \mathrm{~cm}$ ). Each box contained two retractable levers. A response on the active lever resulted in the delivery of a chow or HF pellet ( $45 \mathrm{mg}$ ) and the presentation of a compound cue (light+tone; $5 \mathrm{~s}, 2900 \mathrm{~Hz}$ ), followed by a 25-s timeout period. Responding on the inactive lever had no consequences, but was recorded. Prior to the first session, rats were fasted for $24 \mathrm{~h}$, and placed into the chambers overnight for $14 \mathrm{~h}$ from 1800 to 0800 hours during which time they were able to lever press for either HF or chow rewards. After this session, rats were returned to their home cages for the remainder of the day. Subsequently, rats underwent daily food self-administration training (one $6 \mathrm{~h}$ session per day for 10 days) during which time active lever presses, inactive lever presses, and total pellets received were recorded. Incubation of craving was measured as previously described (Grimm et al, 2011) following 1 or 30 days post training. During this test, no food pellets were available and responding on the previously active lever resulted in the 5-s presentation of the food-paired compound cue followed by a 25-s timeout period.

\section{Quantification of Dendritic Spine Density}

Quantification of dendritic spine density followed methods previously described (Ferrario et al, 2005; Bloss et al, 2011; 
Dingess et al, 2016). Briefly, immediately following incubation testing (1 day or 30 days), rats were anesthetized with isoflurane and killed via cardiac perfusion (200 $\mathrm{ml} 0.9 \%$ saline followed by $300 \mathrm{ml} 1.5 \%$ paraformaldehyde (PFA; in $0.1 \mathrm{M}$ phosphate buffer (PBS)). After washing in PBS, brains were coronally sectioned into $200 \mu \mathrm{m}$ slices with a Leica VT1200S vibratome (Leica, Buffalo Grove, IL) and collected in PBS. Slices were post-fixed (4\% PFA; $20 \mathrm{~min}$ ), incubated with Vybrant-DiI cell-labeling solution $(1: 200$, Invitrogen, Carlsbad, CA; $1 \mathrm{~h}, \mathrm{RT})$, rinsed in PBS, and then placed in PBS $\left(4^{\circ} \mathrm{C}\right.$ for $48 \mathrm{~h}$ ) to allow dye diffusion across membranes. Finally, slices were mounted on glass slides with Vectashield (Vector, Burlingame, CA) and imaged using a Zeiss confocal microscope. Cortical pyramidal cells in the PFC were visually identified by their morphology (Yang et al, 1996). Dendritic spines were then quantified on third- and fourth-order basal dendrites and second- and third-order apical dendrites in output layer $\mathrm{V}$ of the prelimbic medial PFC (PL-mPFC) and IL-mPFC. Layer V of the PFC was chosen for analysis based upon previous findings from our laboratory, indicating a fatinduced decrease in spine density in this region (Dingess et al, 2016) and additionally because other rewarding substances have been shown to alter this deep layer specifically (Aghajanian and Marek, 1999; Mair and Kauer, 2007; Goriounova and Mansvelder, 2012; Kasanetz et al, 2013). Medium spiny neurons (MSNs) in the NAc were visually identified by their morphology (Preston et al, 1980; Wilson and Groves, 1980) and spines were quantified on third- and fourth-order dendrites. In both the PFC and NAc, the terminal $10 \mu \mathrm{m}$ of selected dendrites was analyzed. Spines were classified based on parameters previously described (Bloss et al, 2011; Dingess et al, 2016). Importantly, spines were classified as mushroom type if the ratio of head to neck diameter was $>1.1$ and the maximum head diameter was $>0.4 \mu \mathrm{m}$. In total, 24-56 dendrites were analyzed per dietary group (eight dendrites per animal, four from each hemisphere) for each brain region. Spine analyses were compared to handled, untrained, age-matched controls, and analyses were conducted blind to the experimental condition. Similar to previous studies (Shen et al, 2009; Dingess et al, 2016), statistical analyses were performed by averaging spine types from the eight observed dendritic segments within animals per brain region.

\section{Synaptic Electrophysiology}

To determine whether there was a change in glutamatergic transmission associated with incubation, whole-cell electrophysiology was conducted on coronal slices through the NAc-C immediately after incubation testing. Only one cell per rat was used for each experiment so that reported $n$ values represent the number of animals. Recording conditions and solutions for whole-cell recordings were as previously described (Slaker et al, 2015). All experiments utilized cesium chloride $(\mathrm{CsCl})$ internal solution with spermine $(0.1 \mathrm{mM})$. MSNs within the NAc-C were identified by their morphology. For AMPA/NMDA ratios, neurons were held at $+40 \mathrm{mV}$ in $100 \mu \mathrm{M}$ picrotoxin and $50 \mu \mathrm{M}$ D-(-)-2-amino-5-phosphonopentanoic acid (d-APV) was added once a stable baseline was acquired. Peak AMPAR excitatory postsynaptic current (EPSC) amplitudes were measured at $20-25 \mathrm{~min}$ in d-APV, and this EPSC was subtracted from 5 min averages of baseline EPSCs to obtain the peak NMDA receptor EPSC. Current-voltage $(I-V)$ curves were generated following a stable responding after the addition of d-APV. All drugs and reagents were obtained from Sigma-Aldrich (St Louis, MO).

\section{$\mathrm{BS}^{3}$ Crosslinking and Western Blotting}

Established $\mathrm{BS}^{3}$ crosslinking, SDS-PAGE, and western blotting procedures were used to examine GluA1 and GluA2 surface vs intracellular expression, as in Boudreau et al (2012), at 1 or 30 days after chow self-administration training ( 1 or 30 days) without additional testing for incubation of 'craving.' Behavioral testing was eliminated to get a 'snapshot' of AMPAR expression prior to cue reintroduction as the observed functional changes in rectification $(\sim 10 \%)$ are at the limit of detection for biochemical analysis. Therefore, this measure was used to assess whether there was an available intracellular pool of CP-AMPARs that could be translocated to the synapse if necessary. We focused on GluA1 and GluA2 expression levels as they provide a good indication of changes in CP- and non-CP-AMPAR expression due to the fact that CP-AMPARs lack the GluA2 subunit (Hollmann et al, 1991; Isaac et al, 2007). $\mathrm{BS}^{3}$ crosslinked samples were heated in Laemmli sample treatment buffer with $5 \%$ b-mercaptoethanol $\left(70{ }^{\circ} \mathrm{C}, 10 \mathrm{~min}\right)$, loaded $(20 \mu \mathrm{g}$ protein), and electrophoresed on $4-15 \%$ Bis-Tris gradient gels under reducing conditions. Proteins were transferred onto PVDF membranes (Amersham Biosciences, Piscataway, NJ). Membranes were rinsed, blocked ( $1 \mathrm{~h}, \mathrm{RT}, 5 \%(\mathrm{w} / \mathrm{v})$ nonfat dry milk in TBS-Tween 20 (TBS-T; $0.05 \%$ Tween $20, \mathrm{v} / \mathrm{v})$ ), and incubated overnight $\left(4^{\circ} \mathrm{C}\right)$ with primary antibodies (1:1000 in TBS) to GluA1 (Thermo Scientific; PA1-37776) or GluA2 (NeuroMab, UCDavis/NIH: 75-002). Membranes were washed in TBS-T, incubated with HRP-conjugated secondary (Invitrogen, Carlsbad, CA; $1 \mathrm{~h}$, RT), washed, and immersed in the chemiluminescence detecting substrate (GE Health-care, Piscataway, NJ). Images were acquired on film and Ponceau S (Sigma-Aldrich) was used to determine total protein. Bands of interest were quantified using Image $\mathrm{J}(\mathrm{NIH})$.

\section{Statistical Analysis}

All statistical tests were conducted using Prism 6 (GraphPad Software) using two-way and two-way repeated measures (RMs) ANOVAs as appropriate. When applicable, Sidak's (behavioral, electrophysiological, and biochemical analyses), or Tukey's (spine density) multiple comparison tests were used. All data are shown as mean \pm SEM.

\section{RESULTS}

Both chow and HF groups demonstrated proficiency of the behavioral task, evidenced by the preferential responding to the active $v s$ inactive lever during training (Supplementary Figure S1B and C). Two-way RMs ANOVA revealed a significant day effect and significant diet effect for active lever pressing during testing. Both groups demonstrated an increase in active lever responding (incubation of craving) between 1 and 30 days post training (chow $(n=30) 1$ day: $22.67 \pm 2.71, \quad 30$ days: $64.27 \pm 3.81$; $\operatorname{HF}(n=28) 1$ day: $46.89 \pm 4.69,30$ days: $98.00 \pm 8.83, \mathrm{~F}_{(1,56)}=84.12, p<0.001$, 
Figure $1 \mathrm{~b}$ ); however, the HF group responded significantly more to the active lever than the chow group at both testing time points $\left(\mathrm{F}_{(1,56)}=25.65, p<0.001\right)$ and during training $\left(\mathrm{F}_{(1,779)}=13.51, p<0.001\right.$, Table 1$)$. Unsurprisingly, two-way RMs ANOVA also revealed a significant day and diet effect for cue presentations received during testing $\left(\mathrm{F}_{(1,56)}=86.30\right.$, $p<0.001 ; \mathrm{F}_{(1,56)}=23.28, p<0.001$, respectively, Figure 1d). There were no significant differences in body weight between groups across training or during cue-testing (Figure 1e and Supplementary Figure S1E).

Dendritic spine density and spine type in the PFC and NAc were quantified following incubation of craving testing at 1 and 30 days (Tables 2 and 3). Two-way RMs ANOVA revealed a significant effect of spine type and significant dietary effect on basal and apical dendrites of the IL-PFC at 1 and 30 days. Tukey's multiple comparison's test revealed that rats in the HF group exhibited a significant decrease in thin spine density on basal and apical dendrites of the IL-PFC when compared to naive controls at both 1 day (basal: HF $(n=4): 6.13 \pm 0.22$, naive $(n=3): 7.50 \pm 0.19, p<0.001$, Figure 2d; apical: HF: $6.16 \pm 0.16$, naive: $7.21 \pm 0.11$, $p<0.001$, Figure 2e) and 30 days (basal: HF $(n=6)$ : $6.39 \pm 0.13$, naive $(n=4): 7.59 \pm 0.12, p<0.001$, Figure 2 d; apical: HF: $6.29 \pm 0.17$, naive: $7.44 \pm 0.15, \quad p<0.001$, Figure 2e). There were no significant differences in spine density observed within the prelimbic PFC (PL-PFC; Supplementary Figure S2).

Tukey's multiple comparison's test revealed that, at 1 day, the chow group exhibited an increase in spine density attributed to an increase in thin spines within the NAc-C compared to naive controls (chow $(n=4)$ ): $9.97 \pm 0.08$, naive $(n=3): 9.38 \pm 0.14, p<0.01$, Figure $3 \mathrm{~d})$. This significant trend was also observed in the NAc-Sh (chow $(n=4)$ : $9.81 \pm 0.08$, naive $(n=3): 9.29 \pm 0.04, p<0.01$, Figure $3 e)$. At 30 days, in both the NAc-C and NAc-Sh, the increase in spine density was maintained but was attributed to an increase in mushroom-type spines (NAc-sh: chow $(n=7)$ : $3.02 \pm 0.07$, naive $(n=4): 2.59 \pm 0.11, p<0.01$, Figure 3e; NAc-C: chow $(n=7): 3.07 \pm 0.06$, naive $(n=4): 2.72 \pm 0.16$, $p<0.01$, Figure $3 \mathrm{~d}$ ). Curiously, the HF group did not exhibit any structural changes in the NAc-Sh different from the naive controls at either time point (Figure $3 e$ ). In the NAc-C, however, although there were no observable differences 1 day after training, the HF group showed an increase in mushroom-type spines 30 days following training (HF $(n=6): 3.13 \pm 0.09$, naive $(n=4): 2.72 \pm 0.16, p<0.001$, Figure $3 \mathrm{~d})$.

To further investigate the plasticity in the NAc-C, we utilized whole-cell electrophysiology. Two-way RMs ANOVA revealed that there was no significant dietary effect on AMPA/NMDA ratio $\left(\mathrm{F}_{(1,14)}=0.02, p=0.88\right)$ but that there was a significant day effect $\left(\mathrm{F}_{(1,14)}=14.55, p<0.01\right.$, Figure $4 \mathrm{~b}$ ) such that both groups exhibited an increase in AMPA/NMDA from 1 to 30 days (chow $(n=9) 1$ day: $1.95 \pm 0.21,30$ days: $3.08 \pm 0.27 ; \operatorname{HF}(n=7) 1$ day: $1.86 \pm 0.29$, 30 days: $3.09 \pm 0.37$ ). Interestingly, AMPA EPSCs of MSNs showed a significant increase in rectification index (RI) from 1 to 30 days in chow rats only ( 1 day $(n=8): 0.89 \pm 0.04$, 30 days $(n=6): 1.15 \pm 0.08, p<0.01$, Figure $4 c)$, indicating an increase in CP-AMPA receptor-mediated synaptic transmission. The increase in AMPA/NMDA but the lack of change in RI in HF animals could be interpreted as an increase in
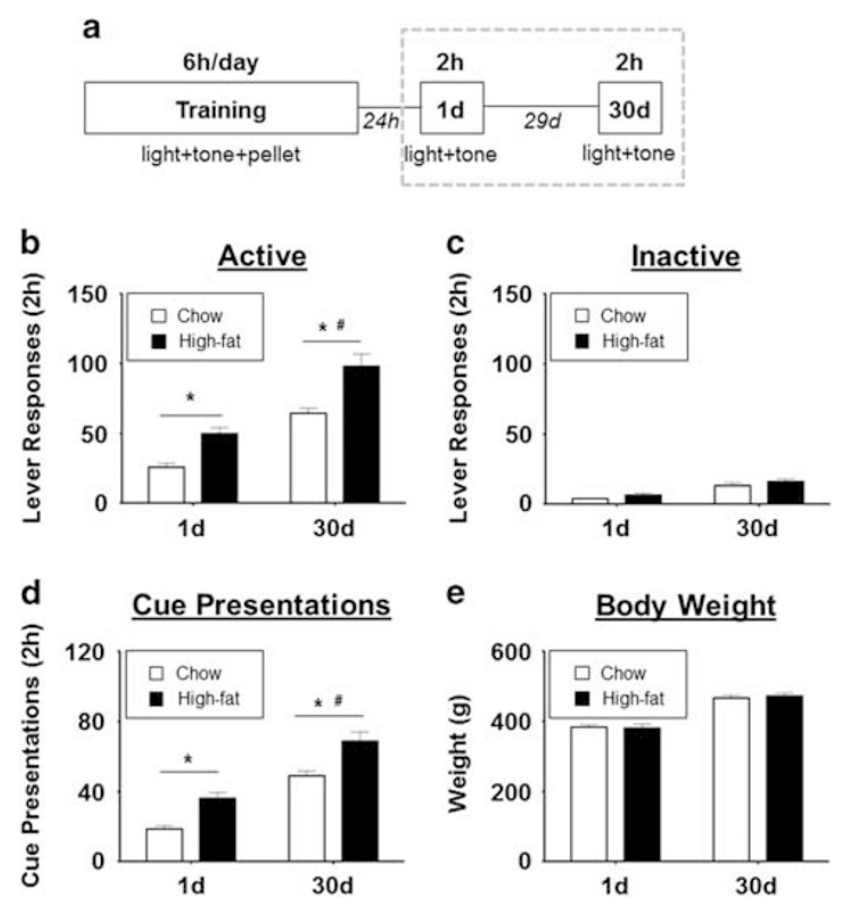

Figure I Both groups exhibit an incubation of craving effect but the response for HF pellets is greater than chow pellets at both I and 30 days post training. (a) Experimental timeline. Gray dotted line indicates portion of timeline from which data for $b-e$ was measured. ( $b$ and $c$ ) Responses toward the active (b) and inactive (c) lever by chow $(n=30$, white) and high-fat ( $n=28$, black) groups during incubation of craving testing $I$ and 30 days post training. (d) Cue presentations received by chow and HF groups during incubation of craving testing I and 30 days post training. (e) Body weights during each cue presentation session. Data reflect only animals that underwent testing at both $\mid$ and 30 days. *Indicates significant difference between chow vs HF. " Indicates significant difference between I and 30 days in both chow and HF groups. Values represent the mean \pm SEM $(p<0.05)$.

Table I Active Lever Responses Throughout Experimental Timeline

\begin{tabular}{lcc}
\hline Day & Chow & HF \\
\hline 1 & $137.0 \pm 16.8$ & $178.7 \pm 20.2$ \\
2 & $101.0 \pm 11.0$ & $98.4 \pm 11.4$ \\
3 & $90.7 \pm 8.2$ & $109.8 \pm 11.3$ \\
4 & $91.8 \pm 7.6$ & $107.6 \pm 10.4$ \\
5 & $107.2 \pm 9.2$ & $127.1 \pm 9.5$ \\
6 & $116.7 \pm 12.0$ & $132.4 \pm 8.8$ \\
7 & $106.4 \pm 9.4$ & $133.0 \pm 12.4$ \\
8 & $123.4 \pm 13.7$ & $134.8 \pm 12.4$ \\
9 & $103.1 \pm 11.5$ & $109.0 \pm 8.9$ \\
10 & $109.2 \pm 11.1$ & $154.6 \pm 16.5 *$ \\
$\mid$ day & $22.7 \pm 2.7$ & $46.9 \pm 4.7 *$ \\
30 days & $64.3 \pm 3.8$ & $98.0 \pm 8.8 *$ \\
\hline
\end{tabular}

Values represent the mean \pm SEM $(* p<0.05)$.

CI-AMPAR-mediated transmission or alternatively a change in NMDA receptor strength (Franks and Isaacson, 2005; Tizabi et al, 2012; Counotte et al, 2014). Indeed, NMDA receptor expression is altered following exposure to both 
Table 2 Summary of Dendritic Spine Quantification Following Cue Reintroduction at I day

\begin{tabular}{|c|c|c|c|c|c|c|}
\hline & \multicolumn{3}{|c|}{ Basal } & \multicolumn{3}{|c|}{ Apical } \\
\hline & Naive & Chow & HF & Naive & Chow & HF \\
\hline Filopodium & $0.29 \pm 0.04$ & $0.33 \pm 0.04$ & $0.22 \pm 0.06$ & $0.29 \pm 0.04$ & $0.21 \pm 0.04$ & $0.22 \pm 0.06$ \\
\hline Thin & $7.42 \pm 0.22$ & $7.50 \pm 0.07$ & $7.53 \pm 0.08$ & $7.29 \pm 0.23$ & $7.38 \pm 0.07$ & $7.47 \pm 0.06$ \\
\hline Branched & $0.25 \pm 0.00$ & $0.21 \pm 0.08$ & $0.22 \pm 0.11$ & $0.25 \pm 0.07$ & $0.13 \pm 0.00$ & $0.13 \pm 0.05$ \\
\hline Total & $12.17 \pm 0.27$ & $12.13 \pm 0.07$ & $12.19 \pm 0.19$ & $11.83 \pm 0.25$ & $11.83 \pm 0.18$ & $11.88 \pm 0.14$ \\
\hline \multicolumn{7}{|l|}{ IL-PFC } \\
\hline Filopodium & $0.29 \pm 0.08$ & $0.29 \pm 0.03$ & $0.31 \pm 0.04$ & $0.29 \pm 0.04$ & $0.17 \pm 0.04$ & $0.34 \pm 0.06$ \\
\hline Branched & $0.17 \pm 0.08$ & $0.17 \pm 0.07$ & $0.16 \pm 0.03$ & $0.13 \pm 0.00$ & $0.13 \pm 0.00$ & $0.19 \pm 0.08$ \\
\hline \multirow[t]{3}{*}{ Total } & $12.08 \pm 0.44$ & $\mid 2.20 \pm 0.11$ & $10.69 \pm 0.15$ & $11.63 \pm 0.22$ & $\mid 1.71 \pm 0.15$ & $10.50 \pm 0.26$ \\
\hline & \multicolumn{3}{|c|}{ Core } & \multicolumn{3}{|c|}{ Shell } \\
\hline & Naive & Chow & HF & Naive & Chow & HF \\
\hline \multicolumn{7}{|c|}{ Nucleus accumbens } \\
\hline Filopodium & $0.21 \pm 0.08$ & $0.31 \pm 0.04$ & $0.33 \pm 0.11$ & $0.21 \pm 0.04$ & $0.34 \pm 0.08$ & $0.29 \pm 0.04$ \\
\hline Thin & $9.38 \pm 0.14$ & $9.97 \pm 0.08$ & $9.50 \pm 0.14$ & $9.29 \pm 0.04$ & $9.81 \pm 0.08$ & $9.38 \pm 0.07$ \\
\hline Stubby & $1.79 \pm 0.11$ & $1.66 \pm 0.06$ & $1.75 \pm 0.07$ & $1.58 \pm 0.11$ & $1.53 \pm 0.06$ & $1.58 \pm 0.11$ \\
\hline
\end{tabular}

Spine quantification in the prelimbic medial prefrontal cortex (PL-mPFC), infralimbic mPFC (IL-mPFC), and nucleus accumbens (NAc), respectively. Bold indicates significant decrease in high-fat (HF)-exposed animals compared to both chow-exposed animals and naive controls. Italics indicate significant increase in chow-exposed animals compared to both HF-exposed animals and naive controls. Values represent the mean \pm SEM $(p<0.05)$.

natural rewards and drugs of abuse, which could account for changes in the AMPA/NMDA ratio (Huang et al, 2009; Brown et al, 2011; Pitchers et al, 2012; Counotte et al, 2014). Although the expression of NMDA receptors was not conclusively studied here, an evaluation of NMDAR decay revealed no significant time or dietary effects, suggesting a lack of change in subunit composition of this receptor (function). This observation, coupled with the results of our spine analysis (Figure 3), suggests that the observed changes in AMPA/NMDA are likely a result of increases in AMPARmediated transmission.

Given that chow groups showed a withdrawal-dependent increase in CP-AMPARs, we next used $\mathrm{BS}^{3}$ crosslinking and western blotting approaches to determine whether AMPAR surface $v s$ intracellular expression is altered following training and withdrawal only. An additional cohort of rats was trained on the chow self-administration task and NAc tissue was collected at $1(n=6)$ or 30 days $(n=8)$ post training without further behavioral testing (no incubation of craving assessment). This allowed us to determine whether
AMPAR surface expression is altered prior to testing. Western blotting for GluA1 and GluA2 showed a timedependent increase in GluA1 (two-way RM ANOVA; main effect of time: $F_{(1,12)}=11.97, p=0.0047$, Figure 5b), but not GluA2 protein expression (data not shown). Interestingly, GluA1 intracellular expression was increased at 30 days (two-way RM ANOVA; sig. time $\times$ location interaction: $\mathrm{F}_{(1,12)}=30.62, \quad p=0.0001$; Sidak's multiple comparison, intracellular 1 vs 30 days $p<0.05$, Figure 5b). This accumulation of intracellular GluA1 in the absence of any changes in GluA2 is consistent with an accumulation of intracellular CP-AMPARs across withdrawal. Taken together, with electrophysiological results above, these biochemical data suggest that CP-AMPARs may be recruited to synapses from this intracellular pool during testing.

\section{DISCUSSION}

The purpose of this study was to determine the neural adaptations associated with the increase in operant 
Table 3 Summary of Dendritic Spine Quantification Following Cue Reintroduction at 30 days

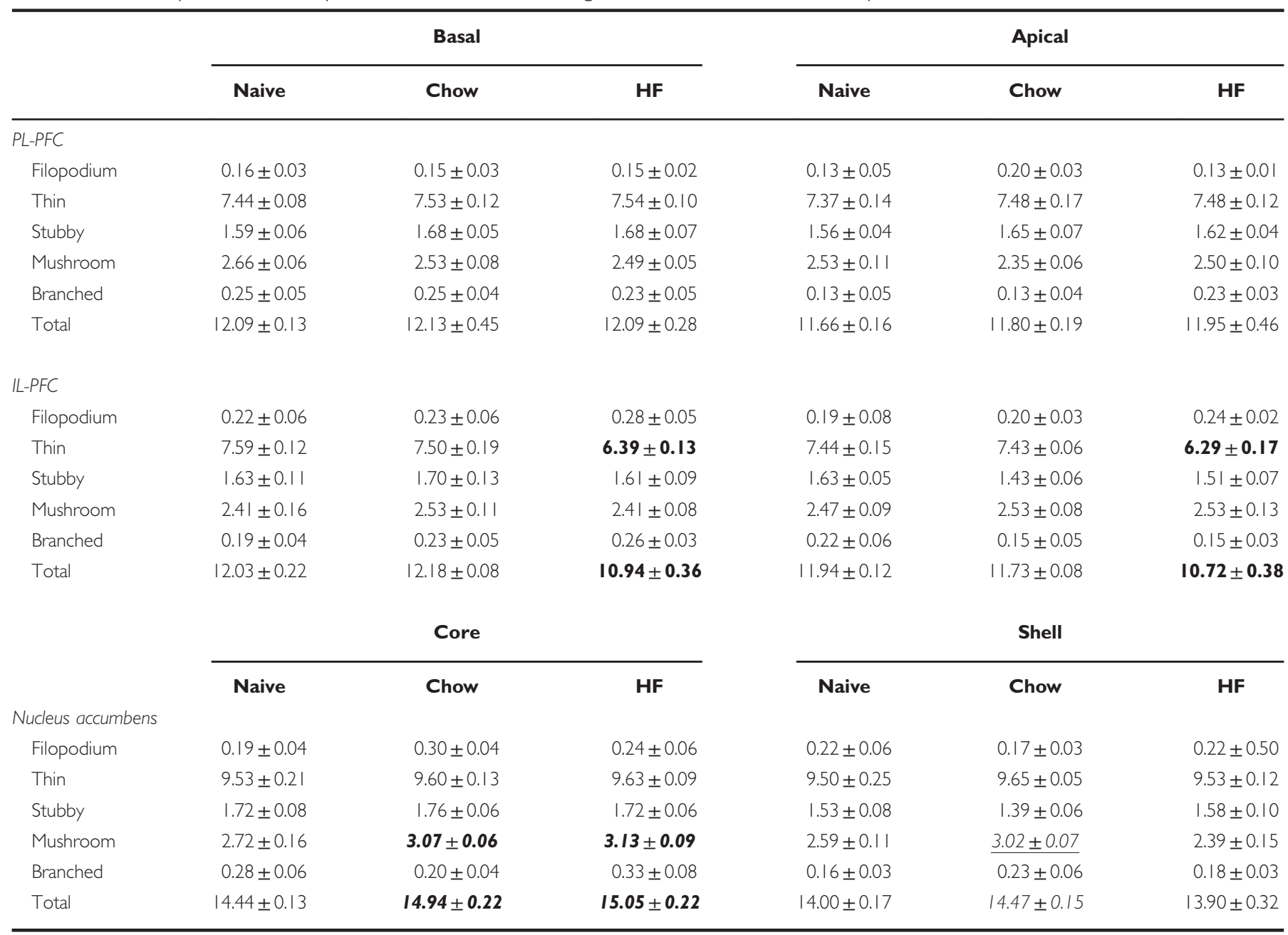

Spine quantification in the prelimbic medial prefrontal cortex (PL-mPFC), infralimbic mPFC (IL-mPFC), and nucleus accumbens (NAc), respectively. Bold indicates significant decrease in high-fat (HF)-exposed animals compared to both chow-exposed animals and naive controls. Underlined italics indicate significant increase in chowexposed animals compared to both HF-exposed animals and naive controls. Bold italics indicate significant increase in both chow- and HF-exposed animals compared to naive controls. Italics indicate chow-exposed animals greater than HF-exposed animals only. Values represent the mean \pm SEM $(p<0.05)$.

responding for food cues following periods of abstinence from the primary reinforcer. In addition, we aimed to assess whether palatability influenced these neural adaptations. Our results demonstrated a time-dependent increase in active lever responding for both chow and HF (incubation of 'craving', Figure 1b). Although the chow pellet was manufactured to mimic the macronutrient composition of the home-cage diet, the incubation of chow craving was not unexpected given that previous studies have indicated that this macronutrient composition is sufficient to induce this phenomenon (Krasnova et al, 2014; Darling et al, 2016). Research from our laboratory indicates that incubation of craving for chow pellets may be the result of novelty as incubation fails to develop when rats have prior experience with the pellets (achieved by rearing rats exclusively on chow pellets rather than the standard lab chow; Darling et al, 2016).

The results of the current study corroborate our previous work that rats display an amount of incubated craving for novel food pellets, suggesting that the incubation of craving model may not always reflect 'craving' or indicate the level of 'addiction' and may rather be inherent to the operant task itself. Thus, we use behavioral responsiveness beyond what is observed for chow pellets as an indicator of reward value. Indeed, although the magnitude of incubation was not different between groups relative to their own baseline, the behavioral response to HF cues was significantly greater than the response to chow cues at both time points (Figure 1b), indicating that the palatability of food enhances the behavioral response to its associated cues. It should be noted that while the chow and HF pellets were distinct in their macronutrient composition, they were similar in their overall caloric density, and thus the greater responsiveness to HF cues is likely due to fat as a macronutrient rather than differences in caloric density. Interestingly, this is different than what we observed in our previous study, which demonstrated that both chow and HF groups trained for $2 \mathrm{~h}$ exhibit equivalent lever responding at both 1 and 30 days (Darling et al, 2016). This supports work within the drug abuse field that extended access paradigms can escalate 
a

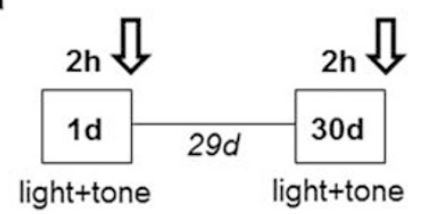

b

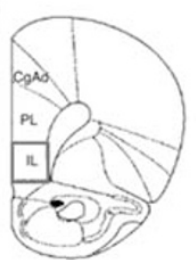

C

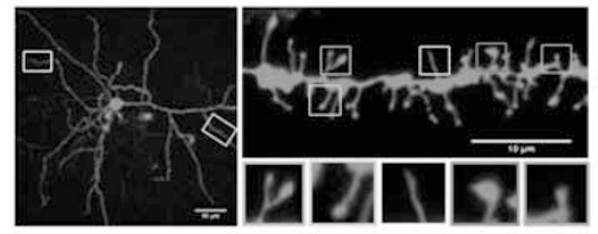

d

BASAL DENDRITES
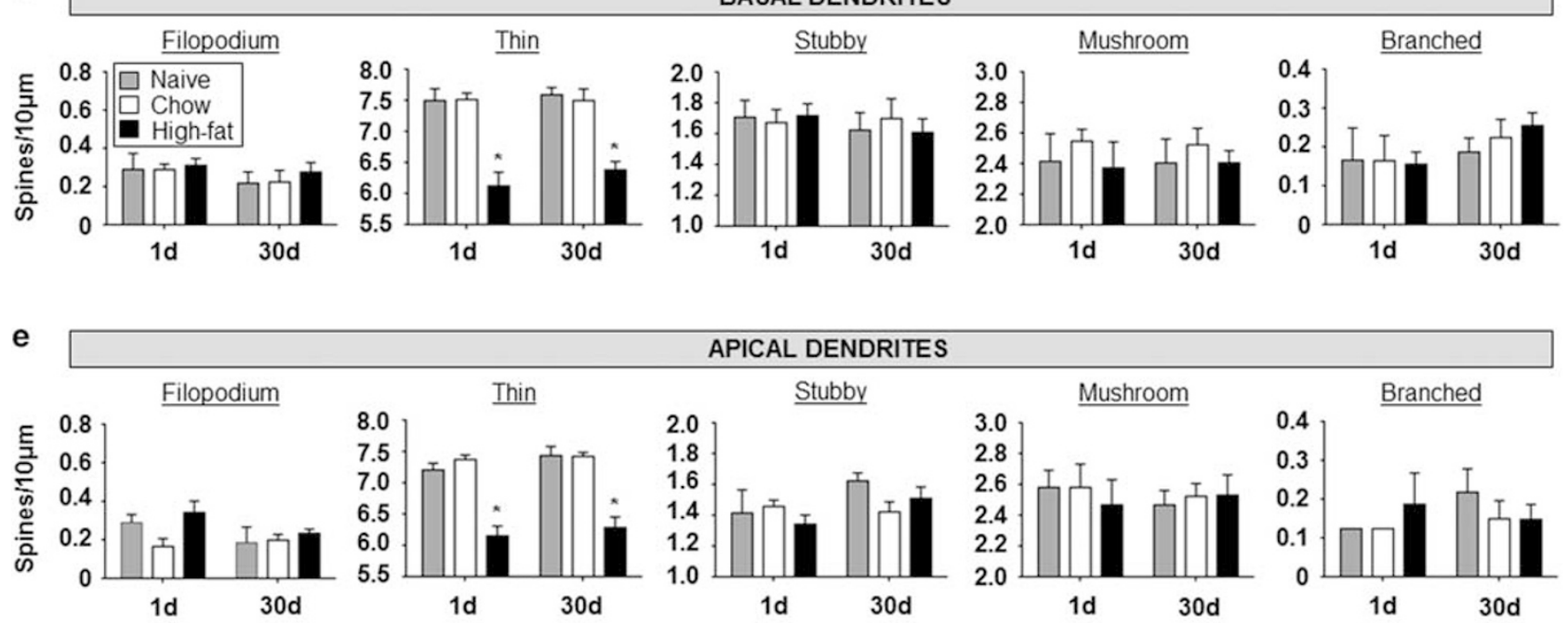

f
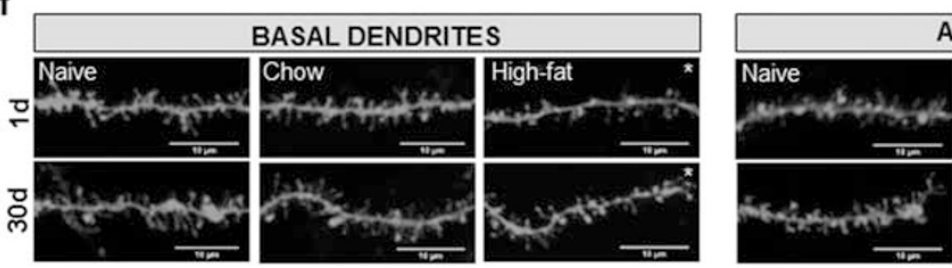

APICAL DENDRITES
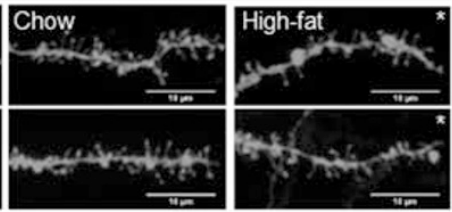

Figure 2 High-fat-exposed animals exhibit a reduction of thin spines in the infralimbic prefrontal cortex (IL-PFC) at both I and 30 days. (a) Experimental timeline. Arrows indicate times at which spine analysis was conducted. (b) Schematic of region analyzed. (c) Representative image of a Dil-stained pyramidal cell in the medial PFC (mPFC). White rectangles indicate example regions where spine analysis on basal and apical dendrites was conducted (left). Example basal dendrite of the mPFC acquired from a naive animal (right). Green, orange, yellow, blue, and purple squares highlight branched-, thin-, filopodium-, mushroom-, and stubby-type spines, respectively. ( $\mathrm{d}$ and e) Quantification of spine types on basal (d) and apical (e) dendrites in the IL-PFC following cue reintroduction at I day (naive (gray), $n=24 / 3$, where the first and second numbers indicate number of dendrites and animals, respectively; chow (white), $n=32 / 8$; high fat (black), $n=32 / 8$ ) and 30 days (naive, $n=32 / 4$; chow, $n=40 / 5$; high fat, $n=48 / 6$ ) post training. (f) Example dendrites from basal (left) and apical (right) dendrites from each dietary condition at I and 30 days. Values represent the mean \pm SEM $(* p<0.05)$. A full color version of this figure is available at the Neuropsychopharmacology journal online.

reward seeking and more accurately recapitulate compulsive use (Wee et al, 2007).

Dendritic spine analyses of the NAc revealed that chow animals exhibited an overall increase in spine density, attributed to an increase in thin-type spines at 1 day and mushroom-type spines at 30 days (Figure $3 \mathrm{~d}$ and e), compared to naive controls. Mushroom spines are considered to be mature synapses that arise in parallel with longterm potentiation (Toni et al, 1999; Yuste and Bonhoeffer, 2001; Matsuzaki et al, 2004) and they contain relatively more AMPARs and AMPA-mediated current than other spine types (Matsuzaki et al, 2001; Bourne and Harris, 2007), making them a likely candidate location for alterations in AMPAR structure and function to occur. Although direct correlations between spine structure and function were not addressed in this study, and changes in spine density do not always parallel changes in AMPA/NMDA (Shen et al, 2011), it is hypothesized that newly formed synapses on thin spines matured from 1 to 30 days post training, correlating with the shift in spine type as well as the observed alterations in AMPA/NMDA ratio (Figure 4). Because of the observation that similar alterations in spine density have been reported following withdrawal from reward in the absence of cue reintroduction (Robinson and Kolb, 1999; Ferrario et al, 2005), we cannot exclude that the observed changes in spine density may be time-dependent rather than cue-dependent. In addition, we cannot rule out that the accumbens associated plasticity, particularly the AMPA/NMDA results, are not the result of the operant procedure itself, independent of reward incentive, as both the chow and HF group showed similar changes. We should point out though that macronutrient profile did have an influence on spine density within the NAc-C and if all the changes could be accounted simply due to operant conditioning we might expect to see 
a

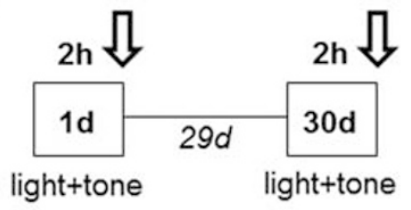

b

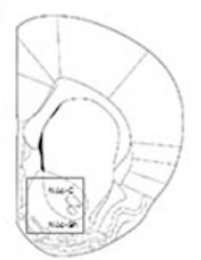

C

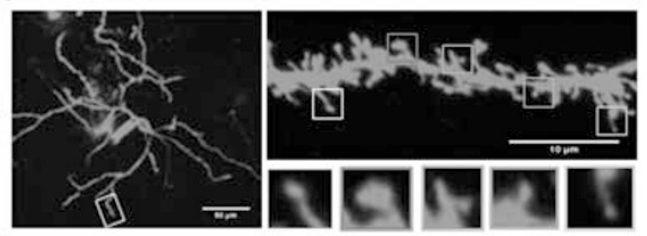

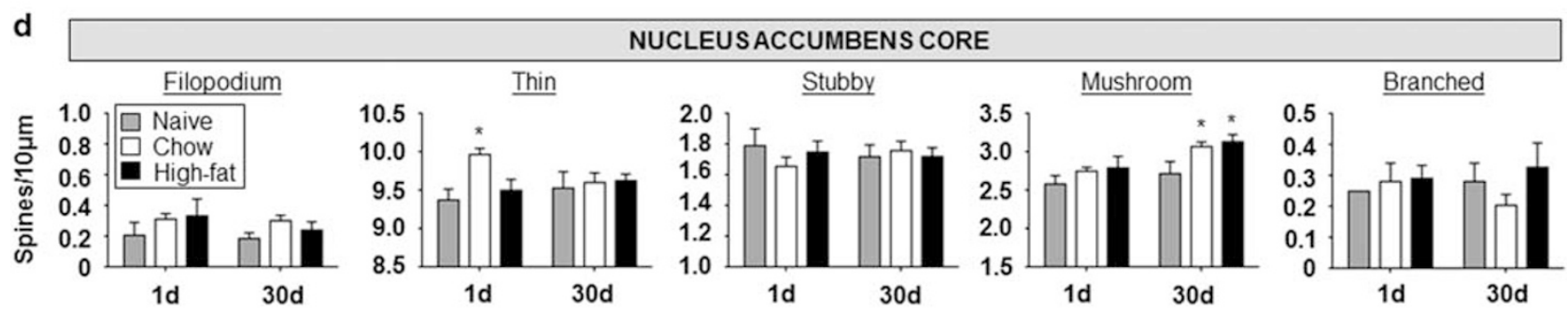
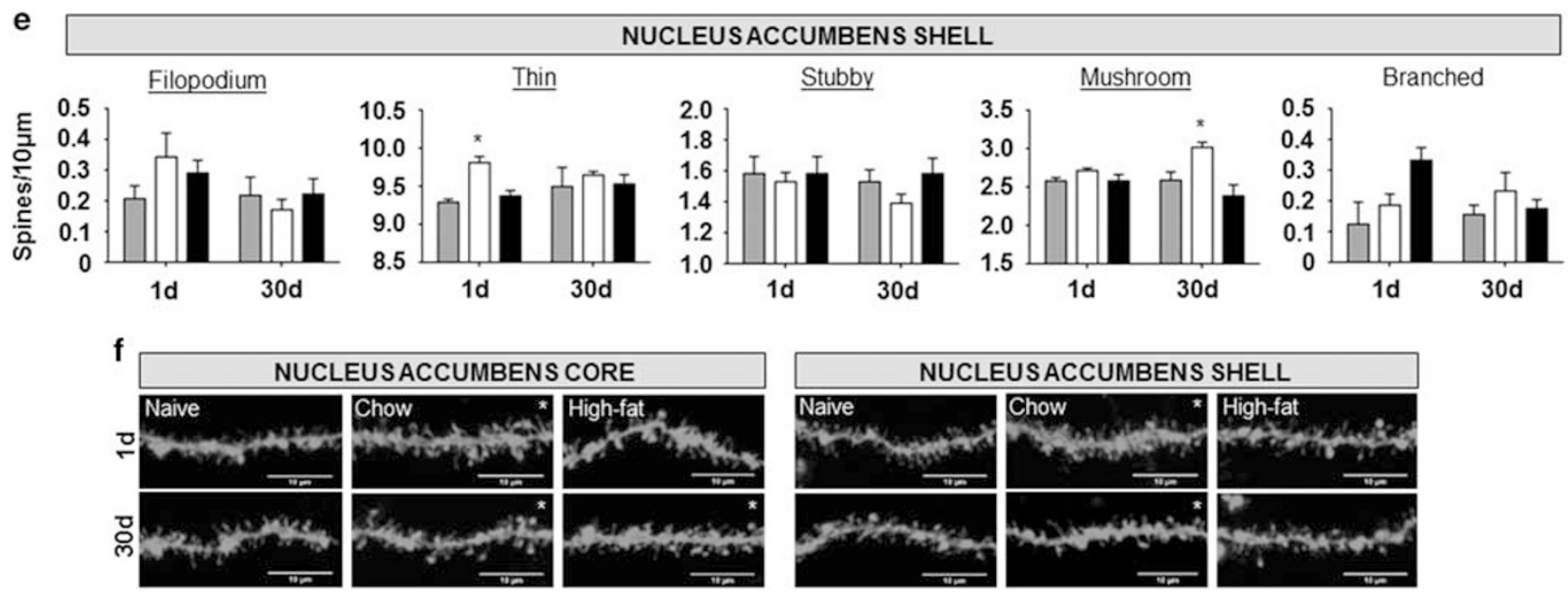

Figure 3 Incubation of chow craving is associated with an increase in thin and mushroom spines in the nucleus accumbens (NAc) at I and 30 days, respectively. (a) Experimental timeline. Arrows indicate times at which spine analysis was conducted. (b) Schematic of region analyzed. (c) Representative image of a Dil-stained medium spiny neuron in the NAc. White rectangle indicates example region where spine analysis on dendrites was conducted (left). Example dendrite of the NAc acquired from a naive animal (right). Yellow, blue, green, purple, and orange squares highlight filopodium-, mushroom-, branched-, stubby-, and thin-type spines, respectively. (d and e) Quantification of spine types from dendrites of the NAc core (d) and NAc shell (e) following cue reintroduction at I day (naive (gray), $n=24 / 3$, where the first and second numbers indicate number of dendrites and animals, respectively; chow (white), $n=32 / 8$; high fat (black), $n=24 / 3$ ) and 30 days (naive, $n=32 / 4$; chow, $n=56 / 7$; high fat, $n=48 / 6$ ) post training. ( $f$ ) Example dendrites of the NAc core (left) and NAc shell (right) from each dietary condition at I and 30 days. Values represent the mean \pm SEM $(* p<0.05)$. A full color version of this figure is available at the Neuropsychopharmacology journal online.

similar spine alterations in both groups immediately following training (1 day). Curiously, despite demonstrating an increase in mushroom spines at 30 days, the HF group failed to exhibit an increase in thin spines at 1 day in the NAc-C (Figure 3). Moreover, the dendritic spine changes we observed in the PFC recapitulated changes we previously saw in animals that were non-contingently exposed to $60 \% \mathrm{HF}$ in their home cages (Dingess et al, 2016), suggesting that the spine alterations in the cortex are independent of learning and rather the result of macronutrient exposure.

In the NAc-Sh, the HF group did not exhibit any changes in spine density compared to naive controls. Although further investigation would be required to decipher the cause of this result, it is possible that the loss of thin-type spines in the IL-PFC, a region that projects to the NAc-Sh, may be a culprit. The staining technique employed in this study however does not allow for the identification of specific output targets and thus cannot be used to answer this question. It is worth noting that based upon our previous research (Dingess et al, 2016), we suspected that fat exposure might alter spine density in the PFC, but it is intriguing that a relatively brief exposure to $\mathrm{HF}$ ( $6 \mathrm{~h}$ daily for 10 days) is sufficient to induce this plasticity independent of obesity, and that it is so long-lasting.

In addition to structural plasticity, we examined functional adaptations in the NAc-C. This region was chosen for analysis because increases in mature mushroom spines were found at 30 days in both chow and HF groups and also because AMPAR transmission in the NAc-C is tightly linked to incubation of reward craving (Conrad et al, 2008; Wolf and Ferrario, 2010; Counotte et al, 2014; Christian et al, 2016). The AMPA/NMDA ratio increased from 1 to 30 days in both the chow and HF groups, consistent with the addition of mature synapses (Figure $4 \mathrm{~b}$ ) and with a role for glutamatergic transmission in the incubation of craving. It should be noted that, although the AMPA/NMDA ratio has 


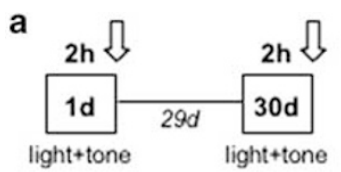

b
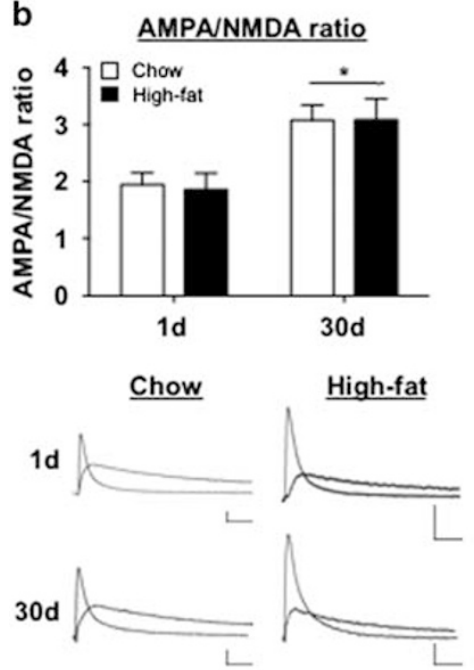

C

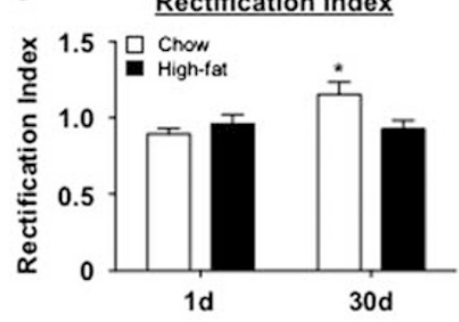

High-fat

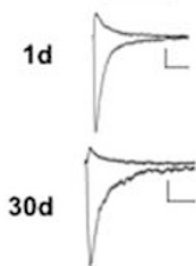

d
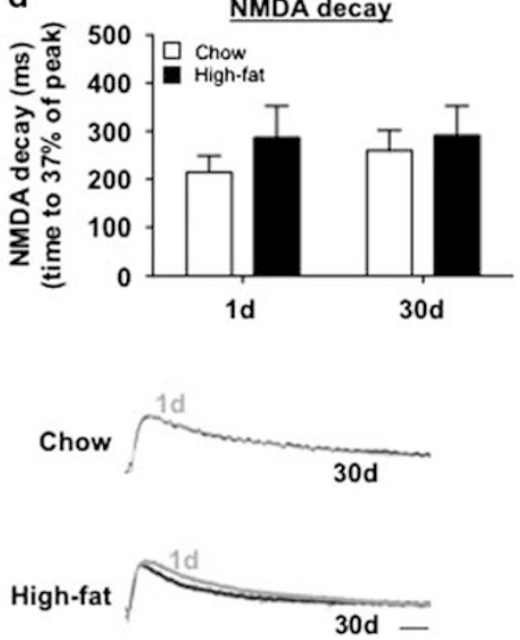

Figure 4 In the NAc-C, incubation of chow and HF craving is associated with an increase in AMPA/NMDA ratio and an increase in CP-AMPARs and Cl-AMPARs, respectively. (a) Experimental timeline. Arrows indicate times at which analysis was conducted. (b) Average $\alpha$-amino-3-hydroxy-5-methyl-4isoxazolepropionic acid (AMPA) to N-methyl-D-aspartate (NMDA) ratio in the nucleus accumbens core (NAc-C) following cue reintroduction I or 30 days post training in chow $(n=9$, white) and high fat $(n=7$, black) groups. Representative AMPA and NMDA traces (below). Vertical scale bar indicates 50 pA; horizontal scale bar indicates $50 \mathrm{~ms}$. (c) Average rectification index of AMPA receptor EPSCs for chow $(n=8)$ and high fat $(n=6)$. Representative traces of AMPA EPSCs (below). (d) NMDA decay. Example traces (below). Values represent the mean \pm SEM $(* p<0.05)$.

a

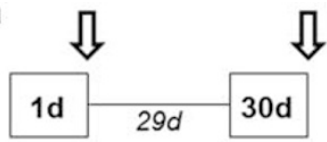

b

\section{Chow GluA1 Expression}

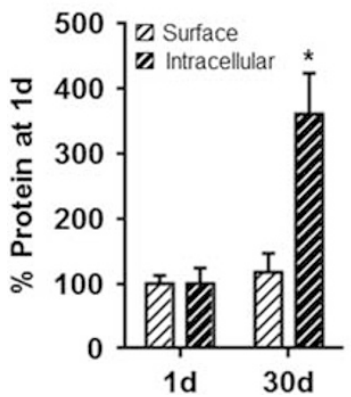

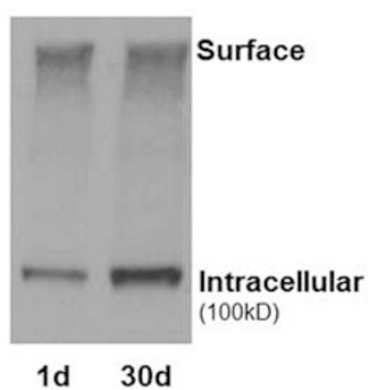

Figure 5 Reintroduction to chow-paired cues elicits an increase in intracellular GluAI expression at 30 days post training. (a) Experimental timeline. Arrows indicate times at which analysis was conducted. (b) GluAI surface (white dashed) and intracellular (black dashed) expression in the NAc (combined core and shell) following I $(n=6)$ or 30 days $(n=8)$ removal from reward and cues (left). Representative western blot (right). Values represent the mean \pm SEM $(* p<0.05)$.

been demonstrated to increase over the developmental period (Hsia et al, 1998), it stabilizes at the onset of adulthood and, thus, the observed increase in AMPA/NMDA is unlikely to be age-dependent and rather be an effect of the incubation of craving for food cues. As all the rats utilized for electrophysiological analysis underwent self-administration training, we cannot rule out that the AMPA/NMDA ratio is elevated at 1 day beyond what might be expected for naive rats. However, the electrophysiological studies were employed specifically to further investigate whether the spinetype shift in the NAc from thin to mushroom (Figure 3) was correlated to functional changes in AMPAR transmission. Naive animals were excluded from this experiment due to their lack of significant change in spine type.

To further investigate the type of AMPA-mediated current involved in the AMPA/NMDA ratio shift, $I-V$ plot analysis was conducted. Interestingly, our results revealed an increase in inwardly rectifying AMPA receptor currents and therefore RI (both hallmarks of CP-AMPARs) from 1 to 30 days in the chow group but not the HF group (Figure 4c). The lack of an increase in CP-AMPAR-mediated transmission in the HF group was curious given that others have demonstrated that exposure to palatable food induces an increase in $\mathrm{CP}$ AMPARs (Tukey et al, 2013; Oginsky et al, 2016) in this region. A key distinction however is that in our study, analysis was conducted following reintroduction to food cues, rather than mere exposure to the food. In fact, our rats had not been exposed to $60 \%$ HF for 30 days.

A separate group of rats was trained to self-administer chow pellets and NAc tissue was collected on 1 or 30 days without additional testing (ie, no cue exposure). Western blotting for GluA1 and GluA2 showed a time-dependent increase in intracellular but not surface expression of GluA1 and no change to GluA2 protein expression (Figure 5b), a result that suggests an increase in the availability of intracellular CP-AMPARs. It is therefore hypothesized that 
the increase in CP-AMPAR-mediated transmission following the expression of incubation of chow craving is the result of rapid synaptic insertion of CP-AMPARs from this intracellular pool in response to testing. This possibility will be addressed in future studies.

In conclusion, for the first time, our results demonstrate reward circuit neuroadaptations that were associated with time-dependent increases in lever responding for chow and HF food rewards (incubation). Although food-associated cues and/or food novelty may drive neuronal adaptations in the NAc that facilitate general food-seeking behavior, we suspect that fat-induced plasticity in the PFC accounts for the robust response to HF cues and ultimately promotes maladaptive feeding. Future studies will examine specific inputs that may be driving this behavior and utilize this knowledge to develop novel therapeutic interventions.

\section{FUNDING AND DISCLOSURE}

We are grateful for the support contributed by the National Institute on Drug Abuse R01DA040965 and the National Institutes of Health Centers Program Grant P30 GM 10339832128 from the National Institute of General Medical Sciences to TEB, PMD, and RAD (University of Wyoming). In addition, the work was supported in part by R01DK106188 to CRF and F31DK111194 to RCD (University of Michigan). The authors declare no conflict of interest.

\section{ACKNOWLEDGMENTS}

We thank Dr Zhaojie Zhang, the director of the Neuroscience microscopy facility at the University of Wyoming, for his help and guidance imaging the spine data. We would also like to thank Kevin Schlidt and Morgan Deters for their assistance with animal care.

\section{REFERENCES}

Abdolahi A, Acosta G, Breslin FJ, Hemby SE, Lynch WJ (2010). Incubation of nicotine seeking is associated with enhanced protein kinase A-regulated signaling of dopamine- and cAMPregulated phosphoprotein of $32 \mathrm{kDa}$ in the insular cortex. Eur J Neurosci 31: 733-741.

Aghajanian GK, Marek GJ (1999). Serotonin and hallucinogens. Neuropsychopharmacology 21: 16S-23S.

Aoyama K, Barnes J, Grimm JW (2014). Incubation of saccharin craving and within-session changes in responding for a cue previously associated with saccharin. Appetite 72: 114-122.

Bienkowski P, Rogowski A, Korkosz A, Mierzejewski P, Radwanska $\mathrm{K}$, Kaczmarek L et al (2004). Time-dependent changes in alcoholseeking behaviour during abstinence. Eur Neuropsychopharmacol 14: $355-360$.

Bloss EB, Janssen WG, Ohm DT, Yuk FJ, Wadsworth S, Saardi KM et al (2011). Evidence for reduced experience-dependent dendritic spine plasticity in the aging prefrontal cortex. J Neurosci 31: 7831-7839.

Boudreau AC, Milovanovic M, Conrad KL, Nelson C, Ferrario CR, Wolf ME (2012). A protein cross-linking assay for measuring cell surface expression of glutamate receptor subunits in the rodent brain after in vivo treatments. Curr Protoc Neurosci Chapter 5: Unit 5.30.1-19.

Bourne J, Harris KM (2007). Do thin spines learn to be mushroom spines that remember? Cur Opin Neurobiol 17: 381-386.
Brown RM, Kupchik YM, Spencer S, Garcia-Keller C, Spanswick DC, Lawrence AJ et al (2015). Addiction-like synaptic impairments in diet-induced obesity. Biol Psychiatry (in press).

Brown TE, Lee BR, Mu P, Ferguson D, Dietz D, Ohnishi YN et al (2011). A silent synapse-based mechanism for cocaine-induced locomotor sensitization. J Neurosci 31: 8163-8174.

Caballero B (2007). The global epidemic of obesity: an overview. Epidemiol Rev 29: 1-5.

Christian DT, Wang X, Chen EL, Sehgal LK, Ghassemlou MN, Miao JJ et al (2016). Dynamic alterations of rat nucleus accumbens dendritic spines over two months of abstinence from extended-access cocaine self-administration. Neuropsychopharmacology 42: 748-756.

Coelho JS, Jansen A, Roefs A, Nederkoorn C (2009). Eating behavior in response to food-cue exposure: examining the cuereactivity and counteractive-control models. Psychol Addict Behav 23: $131-139$.

Conrad KL, Tseng KY, Uejima JL, Reimers JM, Heng LJ, Shaham Y et al (2008). Formation of accumbens GluR2-lacking AMPA receptors mediates incubation of cocaine craving. Nature 454: $118-121$.

Counotte DS, Schiefer C, Shaham Y, O'Donnell P (2014). Timedependent decreases in nucleus accumbens AMPA/NMDA ratio and incubation of sucrose craving in adolescent and adult rats. Psychopharmacology 231: 1675-1684.

Darling RA, Dingess PM, Schlidt KC, Smith EM, Brown TE (2016). Incubation of food craving is independent of macronutrient composition. Sci Rep 6: 30900.

Dingess PM, Darling RA, Kurt Dolence E, Culver BW, Brown TE (2016). Exposure to a diet high in fat attenuates dendritic spine density in the medial prefrontal cortex. Brain Struct Funct 222: 1077-1085.

Eknoyan G (2006). A history of obesity, or how what was good became ugly and then bad. Adv Chronic Kidney Dis 13: 421-427.

Fedoroff IC, Polivy J, Herman CP (1997). The effect of pre-exposure to food cues on the eating behavior of restrained and unrestrained eaters. Appetite 28: 33-47.

Ferrario CR, Gorny G, Crombag HS, Li Y, Kolb B, Robinson TE (2005). Neural and behavioral plasticity associated with the transition from controlled to escalated cocaine use. Biol Psychiatry 58: 751-759.

Fisher JO, Birch LL (2002). Eating in the absence of hunger and overweight in girls from 5 to 7 y of age. Am J Clin Nutr 76: 226-231.

Franks KM, Isaacson JS (2005). Synapse-specific downregulation of NMDA receptors by early experience: a critical period for plasticity of sensory input to olfactory cortex. Neuron 47: 101-114.

Goriounova NA, Mansvelder HD (2012). Nicotine exposure during adolescence leads to short- and long-term changes in spike timing-dependent plasticity in rat prefrontal cortex. J Neurosci 32: 10484-10493.

Grimm JW, Barnes J, North K, Collins S, Weber R (2011). A general method for evaluating incubation of sucrose craving in rats. $J$ Vis Exp 57: e3335.

Grimm JW, Fyall AM, Osincup DP (2005). Incubation of sucrose craving: effects of reduced training and sucrose pre-loading. Physiol Behav 84: 73-79.

Grimm JW, Hope BT, Wise RA, Shaham Y (2001). Neuroadaptation. Incubation of cocaine craving after withdrawal. Nature 412: 141-142.

Harris JL, Bargh JA, Brownell KD (2009). Priming effects of television food advertising on eating behavior. Health Psychol 28: 404-413.

Hollander JA, Carelli RM (2007). Cocaine-associated stimuli increase cocaine seeking and activate accumbens core neurons after abstinence. J Neurosci 27: 3535-3539.

Hollmann M, Hartley M, Heinemann S (1991). Ca2+ permeability of KA-AMPA-gated glutamate receptor channels depends on subunit composition. Science 252: 851-853. 
Hsia AY, Malenka RC, Nicoll RA (1998). Development of excitatory circuitry in the hippocampus. J Neurophysiol 79: 2013-2024.

Huang YH, Lin Y, Mu P, Lee BR, Brown TE, Wayman G et al (2009). In vivo cocaine experience generates silent synapses. Neuron 63: 40-47.

Isaac JT, Ashby MC, McBain CJ (2007). The role of the GluR2 subunit in AMPA receptor function and synaptic plasticity. Neuron 54: 859-871.

Jansen A, Theunissen N, Slechten K, Nederkoorn C, Boon B, Mulkens S et al (2003). Overweight children overeat after exposure to food cues. Eat Behav 4: 197-209.

Jansen A, Vanreyten A, van Balveren T, Roefs A, Nederkoorn C, Havermans R (2008). Negative affect and cue-induced overeating in non-eating disordered obesity. Appetite 51: 556-562.

Kasanetz F, Lafourcade M, Deroche-Gamonet V, Revest JM, Berson $\mathrm{N}$, Balado E et al (2013). Prefrontal synaptic markers of cocaine addiction-like behavior in rats. Mol Psychiatry 18: 729-737.

Koya E, Uejima JL, Wihbey KA, Bossert JM, Hope BT, Shaham Y (2009). Role of ventral medial prefrontal cortex in incubation of cocaine craving. Neuropharmacology 56(Suppl 1): 177-185.

Krasnova IN, Marchant NJ, Ladenheim B, McCoy MT, Panlilio LV, Bossert JM et al (2014). Incubation of methamphetamine and palatable food craving after punishment-induced abstinence. Neuropsychopharmacology 39: 2008-2016.

Lu L, Grimm JW, Hope BT, Shaham Y (2004). Incubation of cocaine craving after withdrawal: a review of preclinical data. Neuropharmacology 47(Suppl 1): 214-226.

Ma YY, Lee BR, Wang X, Guo C, Liu L, Cui R et al (2014). Bidirectional modulation of incubation of cocaine craving by silent synapse-based remodeling of prefrontal cortex to accumbens projections. Neuron 83: 1453-1467.

Mair RD, Kauer JA (2007). Amphetamine depresses excitatory synaptic transmission at prefrontal cortical layer $\mathrm{V}$ synapses. Neuropharmacology 52: 193-199.

Marcelino AS, Adam AS, Couronne T, Koster EP, Sieffermann JM (2001). Internal and external determinants of eating initiation in humans. Appetite 36: 9-14.

Matsuzaki M, Ellis-Davies GC, Nemoto T, Miyashita Y, Iino M, Kasai H (2001). Dendritic spine geometry is critical for AMPA receptor expression in hippocampal CA1 pyramidal neurons. Nat Neurosci 4: 1086-1092.

Matsuzaki M, Honkura N, Ellis-Davies GC, Kasai H (2004). Structural basis of long-term potentiation in single dendritic spines. Nature 429: 761-766.

O'Brien CP, Childress AR, McLellan AT, Ehrman R (1992). Classical conditioning in drug-dependent humans. Ann NY Acad Sci 654: 400-415.

Oginsky MF, Goforth PB, Nobile CW, Lopez-Santiago L, Ferrario CR (2016). Eating 'Junk-Food' produces rapid and long-lasting increases in NAc CP-AMPA receptors; implications for enhanced cue-induced motivation and food addiction. Neuropsychopharmacology 41: 2977-2986.

Ortinski PI, Vassoler FM, Carlson GC, Pierce RC (2012). Temporally dependent changes in cocaine-induced synaptic plasticity in the nucleus accumbens shell are reversed by D1like dopamine receptor stimulation. Neuropsychopharmacology 37: 1671-1682.
Petrovich GD, Ross CA, Gallagher M, Holland PC (2007). Learned contextual cue potentiates eating in rats. Physiol Behav 90: 362-367. Pitchers KK, Schmid S, Di Sebastiano AR, Wang X, Laviolette SR, Lehman MN et al (2012). Natural reward experience alters AMPA and NMDA receptor distribution and function in the nucleus accumbens. PLoS ONE 7: e34700.

Preston RJ, Bishop GA, Kitai ST (1980). Medium spiny neuron projection from the rat striatum: an intracellular horseradish peroxidase study. Brain Res 183: 253-263.

Robinson MJ, Burghardt PR, Patterson CM, Nobile CW, Akil H, Watson SJ et al (2015). Individual differences in cue-induced motivation and striatal systems in rats susceptible to diet-induced obesity. Neuropsychopharmacology 40: 2113-2123.

Robinson TE, Kolb B (1999). Alterations in the morphology of dendrites and dendritic spines in the nucleus accumbens and prefrontal cortex following repeated treatment with amphetamine or cocaine. Eur J Neurosci 11: 1598-1604.

Shen H, Moussawi K, Zhou W, Toda S, Kalivas PW (2011). Heroin relapse requires long-term potentiation-like plasticity mediated by NMDA2b-containing receptors. Proc NAtl Acad Sci USA 108: 19407-19412.

Shen HW, Toda S, Moussawi K, Bouknight A, Zahm DS, Kalivas PW (2009). Altered dendritic spine plasticity in cocainewithdrawn rats. J Neurosci 29: 2876-2884.

Shepard JD, Bossert JM, Liu SY, Shaham Y (2004). The anxiogenic drug yohimbine reinstates methamphetamine seeking in a rat model of drug relapse. Biol Psychiatry 55: 1082-1089.

Slaker M, Churchill L, Todd RP, Blacktop JM, Zuloaga DG, Raber J et al (2015). Removal of perineuronal nets in the medial prefrontal cortex impairs the acquisition and reconsolidation of a cocaine-induced conditioned place preference memory. J Neurosci 35: 4190-4202.

Tizabi Y, Bhatti BH, Manaye KF, Das JR, Akinfiresoye L (2012). Antidepressant-like effects of low ketamine dose is associated with increased hippocampal AMPA/NMDA receptor density ratio in female Wistar-Kyoto rats. Neuroscience 213: 72-80.

Toni N, Buchs PA, Nikonenko I, Bron CR, Muller D (1999). LTP promotes formation of multiple spine synapses between a single axon terminal and a dendrite. Nature 402: 421-425.

Tukey DS, Ferreira JM, Antoine SO, D'Amour JA, Ninan I, Cabeza de Vaca $S$ et al (2013). Sucrose ingestion induces rapid AMPA receptor trafficking. J Neurosci 33: 6123-6132.

Wee S, Specio SE, Koob GF (2007). Effects of dose and session duration on cocaine self-administration in rats. J Pharmacol Exp Ther 320: 1134-1143.

Wilson CJ, Groves PM (1980). Fine structure and synaptic connections of the common spiny neuron of the rat neostriatum: a study employing intracellular inject of horseradish peroxidase. J Comp Neurol 194: 599-615.

Wolf ME, Ferrario CR (2010). AMPA receptor plasticity in the nucleus accumbens after repeated exposure to cocaine. Neurosci Biobehav Rev 35: 185-211.

Yang CR, Seamans JK, Gorelova N (1996). Electrophysiological and morphological properties of layers V-VI principal pyramidal cells in rat prefrontal cortex in vitro. J Neurosci 16: 1904-1921.

Yuste R, Bonhoeffer T (2001). Morphological changes in dendritic spines associated with long-term synaptic plasticity. Ann Rev Neurosci 24: 1071-1089.

Supplementary Information accompanies the paper on the Neuropsychopharmacology website (http://www.nature.com/npp) 